PROCEEDINGS OF THE

AMERICAN MATHEMATICAL SOCIETY

Volume 130, Number 4, Pages 951-957

S 0002-9939(01)06338-9

Article electronically published on November 9, 2001

\title{
INVARIANT IDEALS OF ABELIAN GROUP ALGEBRAS UNDER THE MULTIPLICATIVE ACTION OF A FIELD. II
}

\author{
J. M. OSTERBURG, D. S. PASSMAN, AND A. E. ZALESSKII
}

(Communicated by Lance W. Small)

\begin{abstract}
Let $D$ be a division ring and let $V=D^{n}$ be a finite-dimensional right $D$-vector space, viewed multiplicatively. If $G=D^{\bullet}$ is the multiplicative group of $D$, then $G$ acts on $V$ and hence on any group algebra $K[V]$. In this paper, we completely describe the semiprime $G$-stable ideals of $K[V]$, and conclude that these ideals satisfy the ascending chain condition. As it turns out, this result follows fairly easily from the corresponding results for the field of rational numbers (due to Brookes and Evans) and for infinite locally-finite fields (handled in Part I).
\end{abstract}

\section{INTRODUCTION II}

This is a continuation of [PZ2]. We use the notation of that paper, as well as the material from sections 1 and 2. In particular, this paper begins with section 3 .

We recall that if $V$ is a multiplicative abelian group, then $K[V]$ denotes its group algebra over the field $K$. Furthermore, if $A$ is a subgroup of $V$, then there exists a natural epimorphism $K[V] \rightarrow K[V / A]$ and we let $\omega(A ; V)=\omega_{K}(A ; V)$, the augmentation ideal of $A$ in $V$, denote its kernel. Thus, $\omega(A ; V)$ is the $K$-linear span of all elements of the form $(1-a) v$ with $a \in A$ and $v \in V$. If $G$ is a group which acts as automorphisms on $V$, then $G$ also acts on $K[V]$, and it is clear that $A$ is a $G$-stable subgroup of $V$ if and only if $\omega(A ; V)$ is a $G$-stable ideal of $K[V]$.

Now suppose that $D$ is a division ring and let $V$ be a right $D$-vector space. Then $G=D^{\bullet}$ acts on $V$ and hence on the group algebra $K[V]$, when we view $V$ as a multiplicative group. The goal of this paper is to determine all semiprime $G$-stable ideals of $K[V]$, and our main result is

Theorem B. Let $D$ be an infinite division ring and let $V$ be a finite-dimensional right D-vector space. Furthermore, let $G=D^{\bullet}$ act on $V$ and hence on the group algebra $K[V]$. Then every $G$-stable semiprime ideal of $K[V]$ can be written uniquely as a finite irredundant intersection $\bigcap_{i=1}^{k} \omega\left(A_{i} ; V\right)$ of augmentation ideals, where each $A_{i}$ is a $D$-subspace of $V$. As a consequence, the set of these $G$-stable semiprime ideals is Noetherian.

Received by the editors October 3, 2000.

2000 Mathematics Subject Classification. Primary 16S34, 12E20.

The first author's research was supported by the Taft Committee of the University of Cincinnati. The second author's research was supported in part by NSF Grant DMS-9820271. Much of this work was performed during the third author's visit to the University of Wisconsin-Madison, made possible by the financial support of EPSRC. 
As we will see, this follows fairly easily from two special cases, namely the field of rational numbers (due to Brookes and Evans in $[\mathrm{BE}]$ ) and infinite locally finite fields (handled in Part I). We note that if $D$ is finite, then $D$ is a finite field, and the description of the $G$-stable ideals of $K[V]$ is essentially contained in Lemma 2.1. In particular, there are $G$-stable ideals not contained in the augmentation ideal $\omega(V ; V)$ and there is no uniqueness result. In the case of infinite-dimensional vector spaces, we have at least

Corollary C. Let $D$ be an infinite division ring and let $V$ be a right $D$-vector space of arbitrary dimension. Let $G=D^{\bullet}$ act on $V$ and hence on the group algebra $K[V]$. Then every $G$-stable semiprime ideal of $K[V]$ can be written as an intersection $\bigcap_{i} \omega\left(A_{i} ; V\right)$ of augmentation ideals, where each $A_{i}$ is a D-subspace of $V$.

\section{DIVISION RINGS}

For convenience, we say that a division ring $D$ has property $(*)$ if for any finitedimensional right $D$-vector space $V$ and any field $K$, every $D^{\bullet}$-stable semiprime ideal of $K[V]$ can be written as a finite intersection $\bigcap_{i=1}^{k} \omega\left(A_{i} ; V\right)$ of augmentation ideals, where each $A_{i}$ is a $D$-subspace of $V$. We start with a trivial remark.

Lemma 3.1. Let $D$ be a division ring and let $V$ be a right $D$-vector space.

(i) The group algebra $K[V]$ is semiprime unless char $D=\operatorname{char} K=p>0$.

(ii) If $\operatorname{char} D=\operatorname{char} K=p>0$, then $\omega(V ; V)$ is the unique semiprime ideal of $K[V]$. Hence, this case need not be considered when deciding whether $D$ satisfies property $(*)$.

Proof. If char $D=0$, then $V$ is a torsion-free abelian group. Hence any group algebra $K[V]$ is prime and consequently semiprime. If char $D=p>0$, then $V$ is an elementary abelian $p$-group. In particular, if char $K \neq p$, then $K[V]$ is von Neumann regular, by $[\mathrm{P}$, Theorem 1.1.5], and consequently also semiprime. Finally, if char $K=p$, then $\omega(V ; V)$ is both a nil and a maximal ideal. Hence it is the unique semiprime ideal of the ring. In particular, the $(*)$ condition is automatically satisfied in this case.

Next, we list a few basic observations.

Lemma 3.2. Let $D$ have property $(*)$ and let $V$ be a right $D$-vector space.

(i) $D$ is infinite.

(ii) If $V$ is finite dimensional, then every $D^{\bullet}$-stable semiprime ideal of $K[V]$ can be written uniquely as a finite irredundant intersection $\bigcap_{i=1}^{k} \omega\left(A_{i} ; V\right)$ of augmentation ideals, where each $A_{i}$ is a $D$-subspace of $V$.

(iii) If $V$ has arbitrary dimension, then every $D^{\bullet}$-stable semiprime ideal of $K[V]$ can be written as an intersection $\bigcap_{i} \omega\left(A_{i} ; V\right)$ of augmentation ideals, where each $A_{i}$ is a D-subspace of $V$.

Proof. (i) If $D$ is finite, then so is any finite-dimensional $D$-vector space $V$. If char $K \neq \operatorname{char} D$, then the principal idempotent $e$ of the semisimple group algebra $K[V]$ is centralized by $D^{\bullet}$. Hence the ideal $e K[V]$ is a $D^{\bullet}$-stable semiprime ideal not contained in $\omega(V ; V)$.

(ii) Any finite intersection of augmentation ideals can be reduced to an irredundant intersection. Since $D$ is infinite, all $D^{\bullet}$-sections of $V$ are infinite, so uniqueness follows from Lemma 1.4 . 
(iii) If $I$ is a semiprime ideal of $K[V]$ and if $W$ is any finite-dimensional subspace of $V$, then $I \cap K[W]$ is a semiprime ideal of $K[W]$. Thus, by (*), $I \cap K[W]$ is a finite intersection of augmentation ideals, and Lemma 1.9 yields the result.

Our goal is to prove that every infinite division $\operatorname{ring} D$ satisfies $(*)$. To this end, we first show how to lift this property to certain extensions.

Lemma 3.3. Let $F$ be an infinite field and let $n \geq 1$ be an integer. Then for every integer $k \geq n$, there exists an $n \times k$ matrix $M_{k}$ over $F$ such that all $n \times n$ submatrices of $M_{k}$ are nonsingular.

Proof. It clearly suffices to show that, for each $k \geq 1$, the vector space $F^{n}$ contains a set $S_{k}$ of distinct vectors $\left\{v_{1}, v_{2}, \ldots, v_{k}\right\}$ such that every $n$ of these are linearly independent. We proceed by induction on $k$, starting with a basis $S_{n}$ of $F^{n}$. Then, having found $S_{k}=\left\{v_{1}, v_{2}, \ldots, v_{k}\right\}$, choose $v_{k+1}$ not contained in the union of the finitely many $(n-1)$-dimensional subspaces of $F^{n}$ spanned by the subsets of $S_{k}$ of size $n-1$. Note that, since $F$ is infinite, this union is properly smaller than $F^{n}$. Clearly, $S_{k+1}=\left\{v_{1}, v_{2}, \ldots, v_{k}, v_{k+1}\right\}$ also has the required property.

In the following, we let $F$ be a field and $D$ an $F$-division ring. By this we mean that $F$ is contained in the center of $D$.

Lemma 3.4. Let $D$ be an $F$-division ring, let $V$ be a right $D$-vector space, and assume that $K[V]$ is semiprime. If $F$ has property $(*)$ and if $A$ is an $F$-subspace of $V$, then

$$
\bigcap_{x \in D \bullet} \omega(A ; V)^{x}=\omega(B ; V)
$$

where $B$ is the largest $D$-subspace of $V$ contained in $A$.

Proof. Let $I$ denote the above intersection. Then $I$ is a $D^{\bullet}$-stable ideal and certainly $I \supseteq \omega(B ; V)$. Thus, via the $D^{\bullet}$-homomorphism $K[V] \rightarrow K[V / B]$, it clearly suffices to assume that $B=1$ and then to show that $I=0$. Note that each $\omega(A ; V)^{x}=$ $\omega\left(A^{x} ; V\right)$ is a semiprime ideal by Lemma 3.1(i), and hence $I$ is also a semiprime ideal of $K[V]$.

Suppose, by way of contradiction, that $I \neq 0$. Then we can choose a finitedimensional $F$-subspace $C$ of $V$ with $I \cap K[C] \neq 0$. Thus, since this intersection is a nonzero $F^{\bullet}$-stable semiprime ideal of $K[C]$ and since $F$ has property (*), we can write $I \cap K[C]=\bigcap_{i=1}^{n} \omega\left(C_{i} ; C\right)$, where $C_{1}, C_{2}, \ldots, C_{n}$ are nonidentity $F$-subspaces of $C$. By Lemma 3.2(i), $F$ is infinite.

We first note that for each $y \in D$, there exists $j \in\{1,2, \ldots, n\}$ with $C_{j}^{y} \subseteq A$. This is clear for $y=0$ since $C_{j}^{0}=1$. Now suppose that $y \neq 0$ and note that

$$
\begin{aligned}
\bigcap_{j=1}^{n} \omega\left(C_{j}^{y} ; C^{y}\right) & =(I \cap K[C])^{y}=I^{y} \cap K\left[C^{y}\right]=I \cap K\left[C^{y}\right] \\
& \subseteq \omega(A ; V) \cap K\left[C^{y}\right]=\omega\left(A \cap C^{y} ; C^{y}\right)
\end{aligned}
$$

by Lemma 1.1(i) and the fact that $I$ is $D^{\bullet}$-stable. Thus, since all $F^{\bullet}$-sections on the $F$-space $C^{y}$ are infinite, Lemma 1.4 implies that $\omega\left(C_{j}^{y} ; C^{y}\right) \subseteq \omega\left(A \cap C^{y} ; C^{y}\right)$, for some $j$, and hence that $C_{j}^{y} \subseteq A \cap C^{y} \subseteq A$, as required.

Since $B=1$ and $C_{i} \neq 1$, we know that $\prod_{x \in D} C_{i}^{x} \nsubseteq A$. Thus, for each $i$, there exists $x_{i} \in D^{\bullet}$ with $C_{i}^{x_{i}} \nsubseteq A$. Now take $k=n^{2}$ and form the $n \times n^{2}$ matrix 
$M=\left[f_{r, s}\right]$ over $F$, given by the preceding lemma, with the property that each $n \times n$ submatrix is nonsingular. For each column subscript $s$, let $y_{s}=\sum_{r=1}^{n} x_{r} f_{r, s} \in D$. Then, as we observed above, there exists a subscript $s^{\prime} \in\{1,2, \ldots, n\}$ satisfying $C_{s^{\prime}}^{y_{s}} \subseteq A$. Since there are $n^{2}$ choices for $s$ and only $n$ choices for $s^{\prime}$, it follows that there exist $n$ distinct column subscripts $s$ having the same value $s^{\prime}$. For convenience, let us assume that $C_{1}^{y_{s}} \subseteq A$ for $s=1,2, \ldots, n$.

Let us temporarily revert to additive notation for the $D$-vector space $V$. Then for each $c \in C_{1}$, we have

$$
\sum_{r=1}^{n} c x_{r} f_{r, s}=c y_{s} \in A \quad \text { for all } s=1,2, \ldots, n .
$$

We view this as a system of $n$ linear equations in the $n$ unknowns $c x_{r}$, with matrix of coefficients $N=\left[f_{r, s}\right]$, the first $n$ columns of $M$. Thus, since $\operatorname{det} N \neq 0$ and since $A$ is an $F$-space, we can solve for each $c x_{r}$ and conclude that $c x_{r} \in A$ for all $c \in C_{1}$ and subscripts $r$. In particular, we have $C_{1} x_{1} \subseteq A$ and, since this contradicts the definition of $x_{1}$, we conclude that $I=0$.

One can extend the above result from division rings to certain $F$-algebras containing "mostly" units. Namely, let $R$ be an $F$-algebra and let $V$ be an $R$-module. Then $R^{\bullet}$, the group of units of $R$, acts on $V$ and hence on $K[V]$. To apply the preceding argument to this situation, we need to know that if $x_{1}, x_{2}, \ldots, x_{n} \in R^{\bullet}$, then

$$
\left\{\left(f_{1}, f_{2}, \ldots, f_{n}\right) \in F^{n} \mid f_{1} x_{1}+f_{2} x_{2}+\cdots+f_{n} x_{n} \in R^{\bullet}\right\}
$$

is not contained in a finite union of proper subspaces of $F^{n}$. For example, this holds if $R$ is semilocal, that is, a ring having just finitely many maximal one-sided ideals.

Lemma 3.5. Let $D$ be an F-division ring. If $F$ has property (*), then so does $D$.

Proof. Let $V$ be a finite-dimensional right $D$-vector space and let $I$ be a $D^{\bullet}$-stable semiprime ideal of $K[V]$. In view of Lemma 3.1(i)(ii), it suffices to assume that $K[V]$ is semiprime. Since $I$ is $F^{\bullet}$-stable, it follows from (*) and Lemma 3.2(iii) that $I$ is an intersection of augmentation ideals of the form $\omega(A ; V)$ with $A$ an $F$ subspace of $V$. But $I$ is $D^{\bullet}$-stable, so $I$ can be written as an intersection of ideals of the form $\bigcap_{x \in D} \cdot \omega(A ; V)^{x}$. By Lemma 3.4, each of these latter intersections is an augmentation ideal $\omega(B ; V)$ with $B$ a $D$-subspace of $V$. Thus $I$ is an intersection of suitable $\omega(B ; V)$. Finally, since $\operatorname{dim}_{D} V<\infty$, we see that $V$ has a finite-length composition series as a $D^{\bullet}$-module. With this, we can conclude from Lemma 1.6 that $I$ is a finite intersection $\bigcap_{i=1}^{n} \omega\left(C_{i} ; V\right)$, where each $C_{i}$ is a $D$-subspace of $V$.

Next, we show that property $(*)$ is inherited by division subalgebras. Part (ii) of the following is almost entirely notation.

Lemma 3.6. Let $D \subseteq E$ be division rings.

(i) If $S$ is a finite subset of $E^{\bullet}$ and if $D$ is infinite, then there exists a $D$-linear functional $\lambda:{ }_{D} E \rightarrow{ }_{D} D$ such that $\lambda(1)=1$ and $\lambda(S) \subseteq D^{\bullet}$.

(ii) Let $V$ be a right $D$-vector space and let $W=V \otimes_{D} E$ be the natural extension of $V$ to a right E-vector space. If $\lambda:{ }_{D} E \rightarrow{ }_{D} D$ is a D-linear functional with $\lambda(1)=1$, then the map $\Lambda: W \rightarrow V$ given by $v \otimes e \mapsto v \lambda(e)$ is a group homomorphism which is the identity on $V=V \otimes 1$. Hence $\Lambda$ determines an algebra homomorphism $\Lambda: K[W] \rightarrow K[V]$ which is the identity on $K[V]$. Furthermore, $\Lambda\left(\alpha^{e}\right)=\alpha^{\lambda(e)}$ if $\alpha \in K[V], e \in E^{\bullet}$ and $\lambda(e) \in D^{\bullet}$. 
Proof. (i) We can assume that $1 \in S$. Now $H=\operatorname{Hom}\left({ }_{D} E,{ }_{D} D\right)$ is a right $D$-vector space and, for each $s \in S, H_{s}=\{\lambda \in H \mid \lambda(s)=0\}$ is a proper $D$-subspace. Since $D$ is infinite, $\left|H: H_{s}\right|=\infty$ and it follows from [P] Lemma 4.2.1] that we can choose $\mu \in H$ with $\mu \notin \bigcup_{s \in S} H_{s}$. Thus $\lambda=\mu \cdot \mu(1)^{-1}$ has the required property.

(ii) We note that $W=V \otimes_{D} E$ is the natural extension of $V$ to a right $E$ vector space and we identify $V$ with $V \otimes 1$. Since $\lambda:{ }_{D} E \rightarrow{ }_{D} D$ is a $D$-linear functional, the map $V \times E \rightarrow V$ given by $(v, e) \mapsto v \lambda(e)$ is bilinear and balanced. Hence, it determines an additive homomorphism $\Lambda: W=V \otimes E \rightarrow V$ given by $\Lambda(v \otimes e)=v \lambda(e)$. Then $\Lambda$ is the identity on $V=V \otimes 1$ since $\lambda(1)=1$, and in multiplicative notation, $\Lambda$ determines an algebra homomorphism $\Lambda: K[W] \rightarrow K[V]$ which is the identity on $K[V]$. Finally, if $e \in E^{\bullet}$ and $\lambda(e) \in D^{\bullet}$, then $\Lambda(v e)=$ $\Lambda((v \otimes 1) e)=\Lambda(v \otimes e)=v \lambda(e)$, and this translates in multiplicative language to $\Lambda\left(v^{e}\right)=v^{\lambda(e)}$. Consequently, $\Lambda\left(\alpha^{e}\right)=\alpha^{\lambda(e)}$ for all $\alpha \in K[V]$.

With this, we can prove

Lemma 3.7. Let $D \subseteq E$ be infinite division rings.

(i) Let $V$ be a right $D$-vector space and let $W=V \otimes_{D} E$ be its natural extension to a right E-vector space. Suppose $I$ is a $D^{\bullet}$-stable ideal of $K[V]$ and let $J=\sum_{e \in E^{\bullet}} I^{e} K[W]$ be the $E^{\bullet}$-stable ideal of $K[W]$ generated by $I$. Then $J \cap K[V]=I$ and $\sqrt{J} \cap K[V]=\sqrt{I}$.

(ii) If $E$ has property (*), then so does $D$.

Proof. (i) Certainly, $J \cap K[V] \supseteq I$. For the reverse inclusion, let $\alpha \in J \cap K[V]$ and note that $\alpha \in J$ can be written as a finite sum $\alpha=\sum_{i=1}^{k} \beta_{i}^{e_{i}} \gamma_{i}$ with $\beta_{i} \in I$, $\gamma_{i} \in K[W]$ and $e_{i} \in E^{\bullet}$. Since $D$ is infinite, Lemma 3.6(i) implies that there exists a $D$-linear functional $\lambda:{ }_{D} E \rightarrow{ }_{D} D$ with $\lambda(1)=1$ and $\lambda\left(e_{i}\right) \in D^{\bullet}$ for all $i=1,2, \ldots, k$. We use $\lambda$ in Lemma 3.6(ii) to construct an algebra homomorphism $\Lambda: K[W] \rightarrow K[V]$ which is the identity on $K[V]$ and which satisfies $\Lambda\left(\tau^{e}\right)=\tau^{\lambda(e)}$ for all $\tau \in K[V]$ and all $e \in E^{\bullet}$ with $\lambda(e) \in D^{\bullet}$. Then, by applying $\Lambda$ to the formula for $\alpha$, we obtain

$$
\alpha=\Lambda(\alpha)=\sum_{i=1}^{k} \Lambda\left(\beta_{i}^{e_{i}}\right) \Lambda\left(\gamma_{i}\right)=\sum_{i=1}^{k} \beta_{i}^{\lambda\left(e_{i}\right)} \Lambda\left(\gamma_{i}\right)
$$

since $\lambda\left(e_{i}\right) \neq 0$. But $\beta_{i} \in I$ and $I$ is $D^{\bullet}$-stable, so $\beta_{i}^{\lambda\left(e_{i}\right)} \in I$ and hence $\alpha \in I$.

Finally, it is clear that $\sqrt{J} \cap K[V] \supseteq \sqrt{I}$. For the reverse inclusion, let $\delta \in$ $\sqrt{J} \cap K[V]$. Then for some integer $n>0$, we have $\delta^{n} \in J \cap K[V]=I$, so $\delta \in \sqrt{I}$.

(ii) Let $K$ be a field, let $V$ be a finite-dimensional right $D$-vector space, and let $I$ be a $D^{\bullet}$-stable semiprime ideal of $K[V]$. Set $W=V \otimes_{D} E$ and use the notation of part (i). Then $W$ is a finite-dimensional right $E$-vector space containing $V=V \otimes 1$, and we let $J=\sum_{e \in E} I^{e} K[W]$ be the $E^{\bullet}$-stable ideal of $K[W]$ generated by $I$. Since $\sqrt{J}$ is clearly an $E^{\bullet}$-stable semiprime ideal of $K[W],(*)$ implies that $\sqrt{J}=\bigcap_{i=1}^{k} \omega\left(B_{i} ; W\right)$ is a finite intersection of augmentation ideals with each $B_{i}$ an $E$-subspace of $W$. But $I$ is a semiprime ideal, so $I=\sqrt{I}=K[V] \cap \sqrt{J}$ by (i), and Lemma 1.1(i) yields

$$
I=\sqrt{I}=K[V] \cap \sqrt{J}=K[V] \cap \bigcap_{i=1}^{k} \omega\left(B_{i} ; W\right)=\bigcap_{i=1}^{k} \omega\left(B_{i} \cap V ; V\right) .
$$

Since $B_{i} \cap V$ is a $D$-subspace of $V$, we see that $I$ has the appropriate form. 
It is now a simple matter to obtain our main results.

Proof of Theorem $B$ and Corollary $C$. Let $D$ be an infinite division ring. Our first goal is to show that $D$ has property $(*)$. If char $D=0$, then $D$ contains the field $Q$ of rational numbers as a central subfield, and $Q$ satisfies $(*)$ by $[\mathrm{BE}$, Proposition 6 . Thus, by Lemma 3.5, $D$ has property $(*)$, and it now suffices to assume that $D$ has characteristic $p>0$.

Let $F$ be an infinite field of characteristic $p$ and let $\tilde{F}$ be its algebraic closure. Then $\tilde{F}$ contains $L$, the algebraic closure of $\mathrm{GF}(p)$, and $L$ is an infinite locally finite field. Thus, by Theorem A and Lemma 3.1(ii), we see that $L$ has property $(*)$, and then Lemma 3.5 implies that $\tilde{F}$ has property $(*)$. But $F$ is infinite, so we can now conclude from Lemma 3.7(ii) that $F$ also satisfies $(*)$.

In particular, if $D$ has an infinite center $F$, then $F$ satisfies $(*)$ and therefore so does $D$ by Lemma 3.5. Finally, if $D$ is arbitrary, let $E=D\left[\left[t, t^{-1}\right]\right]$ be the ring of all Laurent series in the variable $t$ with coefficients in $D$. Then $E$ is a division ring, $E \supseteq D$ and $E$ has an infinite center. As we observed, the latter implies that $E$ has property $(*)$, and consequently so does $D$ by Lemma 3.7(ii).

In other words, we have now shown that all infinite division rings $D$ satisfy (*). In particular, by Lemma 3.2(ii), if $V$ is a finite-dimensional right $D$-vector space, then every $D$-stable semiprime ideal of $K[V]$ is uniquely a finite irredundant intersection of augmentation ideals. Furthermore, by Lemma 1.8, the set of all such ideals satisfies the ascending chain condition. Thus Theorem B is proved, and Corollary C is an immediate consequence of Theorem B and Lemma 3.2(iii).

Let $\mathfrak{G}$ be a group acting on a set $\mathcal{S}$. Then we recall that an element $\sigma \in \mathcal{S}$ is said to be $\mathfrak{G}$-orbital if it has finitely many $\mathfrak{G}$-conjugates or equivalently if the stabilizer of $\sigma$ in $\mathfrak{G}$ has finite index in the group.

Corollary 3.8. Let $D$ be an infinite division ring and let $V$ be a finite-dimensional right $D$-vector space, viewed multiplicatively. Suppose $\mathfrak{G}$ is a group which acts on $V$ and contains $D^{\bullet}$, in its natural action, as a normal subgroup. Then every $\mathfrak{G}$-stable semiprime ideal of $K[V]$ is uniquely an irredundant intersection $\bigcap_{A \in \mathfrak{A}} \omega(A ; V)$, where $\mathfrak{A}$ is a finite $\mathfrak{G}$-stable set of $D$-subspaces of $V$. In particular, each $A \in \mathfrak{A}$ is a $D^{\bullet}$-stable, $\mathfrak{G}$-orbital subgroup of $V$.

Proof. If $I$ is $\mathfrak{G}$-stable, then it is $D^{\bullet}$-stable. Hence by Theorem B, $I$ can be written uniquely as the irredundant intersection $I=\bigcap_{i=1}^{m} \omega\left(A_{i} ; V\right)$, where each $A_{i}$ is $D^{\bullet}$ stable. In particular, if $g \in \mathfrak{G}$, then since $I$ is $\mathfrak{G}$-stable, we have

$$
\bigcap_{i=1}^{m} \omega\left(A_{i} ; V\right)=I=I^{g}=\bigcap_{i=1}^{m} \omega\left(A_{i}^{g} ; V\right) .
$$

But $D^{\bullet}$ is normal in $\mathfrak{G}$, so each $A_{i}^{g}$ is certainly a $D^{\bullet}$-stable subgroup of $V$. Hence, by uniqueness, $\left\{A_{1}^{g}, A_{2}^{g}, \ldots, A_{m}^{g}\right\}=\left\{A_{1}, A_{2}, \ldots, A_{m}\right\}$ and consequently $\mathfrak{G}$ permutes the finite set $\mathfrak{A}=\left\{A_{1}, A_{2}, \ldots, A_{m}\right\}$.

This observation yields the following analog of results in $[\mathrm{BE}]$ and $[\mathrm{FS}]$.

Example 3.9. Let $D$ be an infinite division ring, let $V=D^{n}$ and take $\mathfrak{G}=$ $\operatorname{GL}_{n}(D) \cdot D^{\bullet}$, where $D^{\bullet}$ acts on the right on $V$ and $\mathrm{GL}_{n}(D)$ acts on the left. Then $\omega(V ; V)$ is the unique proper $\mathfrak{G}$-stable semiprime ideal of $K[V]$. 
Proof. If $I$ is a proper $\mathfrak{G}$-stable semiprime ideal of $K[V]$, then Corollary 3.8 implies that $I=\bigcap_{A \in \mathfrak{A}} \omega(A ; V)$, where each $A \in \mathfrak{A}$ is a right $D$-subspace of $V$ orbital under the action of $\mathfrak{G}$. But all $\mathfrak{G}$-orbits of proper subspaces of $V$ are clearly infinite, so this implies that $A=1$ or $V$ and, since $I$ is proper, we have $I=\omega(V ; V)$.

As we observed earlier, if $\operatorname{char} D>0$ and $\operatorname{char} K \neq \operatorname{char} D$, then $K[V]$ is von Neumann regular, and hence each ideal is semiprime. In particular, the above shows that $\omega(V ; V)$ is the unique proper $\mathfrak{G}$-stable ideal of $K[V]$. Next, suppose that char $D=0$ and that char $K=q>0$. If $I$ is a proper $\mathfrak{G}$-stable ideal of $K[V]$, then, by considering the radical $\sqrt{I}$, we see that $\omega(V ; V) / I$ is nil. Thus, if $x \in V$, then $1^{q^{n}}-x^{q^{n}}=(1-x)^{q^{n}} \in I$ for some $n>0$ and, since $I$ is closed under the action of the rationals on $V$, we see that $1-x \in I$. Again, this yields $I=\omega(V ; V)$. Lastly, if char $D=$ char $K=0$, then $\omega(V ; V)^{2}$ is properly contained in $\omega(V ; V)$, so there are certainly other $\mathfrak{G}$-stable ideals of $K[V]$ in this case.

\section{ADDED IN PROOF}

When the division ring $D$ and the field $K$ have different characteristics, the semiprime hypotheses in Theorem B and Corollary $\mathrm{C}$ are not really necessary. A quick proof of this observation will appear in a future addendum to this paper.

\section{REFERENCES}

[BE] C. J. B. Brookes and D. M. Evans, Augmentation modules for affine groups, Math. Proc. Cambridge Philos. Soc. 130 (2001), 287-294. CMP 2001:06

[FS] D. R. Farkas and R. L. Snider. Simple augmentation modules, Quart. J. Math. Oxford (2) 45 (1994), 29-42. MR 95d:16032

[P] D. S. Passman, The Algebraic Structure of Group Rings, Wiley-Interscience, New York, 1977. MR 81d:16001

[PZ2] D. S. Passman and A. E. Zalesskiı̌, Invariant ideals of abelian group algebras under the multiplicative action of a field, I, Proc. AMS, to appear.

Department of Mathematics, University of Cincinnati, Cincinnati, Ohio 45221

E-mail address: James.Osterburg@math.uc.edu

Department of Mathematics, University of Wisconsin, Madison, Wisconsin 53706

E-mail address: Passman@math.wisc.edu

School of Mathematics, University of East Anglia, Norwich NR4 7TJ, United KingDOM

E-mail address: A.Zalesskii@uea.ac.uk 